\title{
Mixed infection with two types of echinococcosis misdiagnosed as hepatic alveolar echinococcosis
}

\author{
Qiancheng $\mathrm{Du}^{[1]^{*}}$, Chenliang $\mathrm{Hu}^{[2]^{*}}$ and Yanfei $\mathrm{Li}^{[2]}$ \\ [1]. Department of General Surgery, Shanghai Fourth People's Hospital Affiliated to Tongji University School of Medicine, Shanghai, China. \\ [2]. Department of Hepatopancreatobiliary Surgery, the Affiliated Hospital of Qinghai University \\ and Qinghai Province Key Laboratory of Hydatid Disease Research, Qinghai University, Xining, China.
}

A 40-year-old female visited our hospital with a chief complaint of pain in the right superior abdominal quadrant for the last 2 months. The serological tests were positive for Echinococcosis IgG antibodies. Contrast-enhanced computed tomography of the abdomen revealed multiple irregular lesions in the liver, with a maximum cross-sectional area of $17.4 \times 10.8$ $\mathrm{cm}^{2}$ involving mainly the right lobe and partiallysegments 3 and 4 (Figure A). The preoperative diagnosis was hepatic alveolar

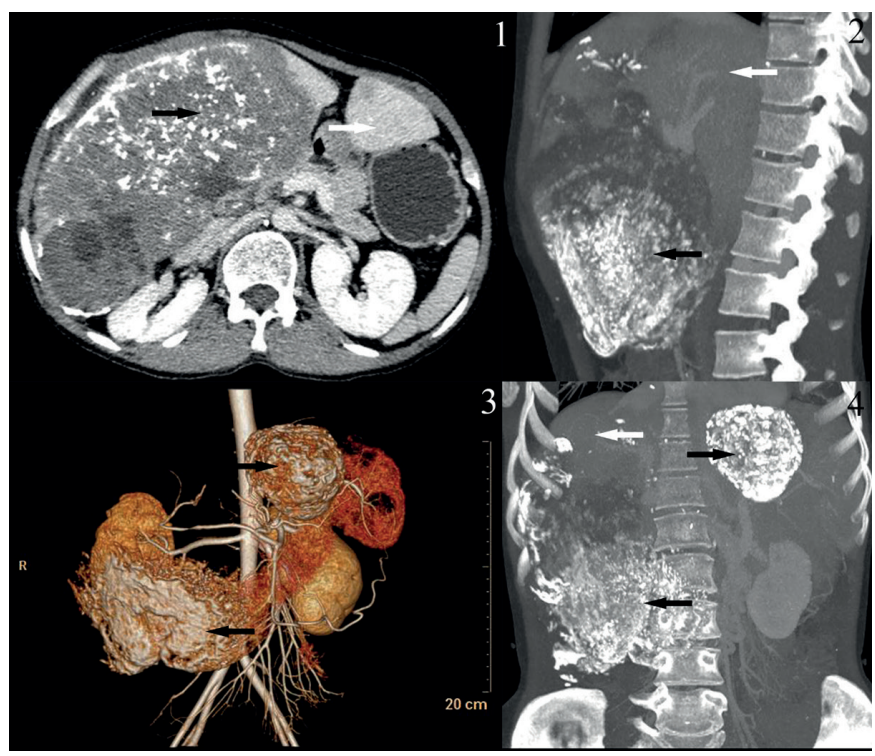

FIGURE A: Abdominal computed tomography examination. The black arrows indicate hepatic echinococcosis lesions; the white arrows indicate normal liver tissue.

${ }^{*}$ contributed equally to this work. Corresponding author: Yanfei Li. e-mail: liyanfei2019vip@163.com Orcid: 0000-0002-8841-6152

Received 05 June 2019

Accepted 18 July 2019 echinococcosis $(\mathrm{P} 4, \mathrm{~N} 1, \mathrm{M} 0)$ based on the typical imaging characteristics, serological test results, medical history, and contact history of the epidemic area, among other factors ${ }^{1}$.

The patient underwent hepatectomy after full preoperative preparation. Upon separation of the lesion from the normal liver tissue, hepatic cystic echinococcosis were unexpectedly found next to the hepatic alveolar echinococcosis (Figure B: 1 and 2). Hydrocortisone (100 mg) was administered approximately 30 minutes before surgery to prevent cystic fluid allergy according to the WHO Informal Working Group on Echinococcosis recommendations for the management of human cystic echinococcosis ${ }^{2}$. Fortunately, the lesion was almost completely calcified and there was no allergic reaction during the operation. Postoperative anatomopathological findings confirmed hepatic echinococcosis (Figure B: 3 and 4). A review

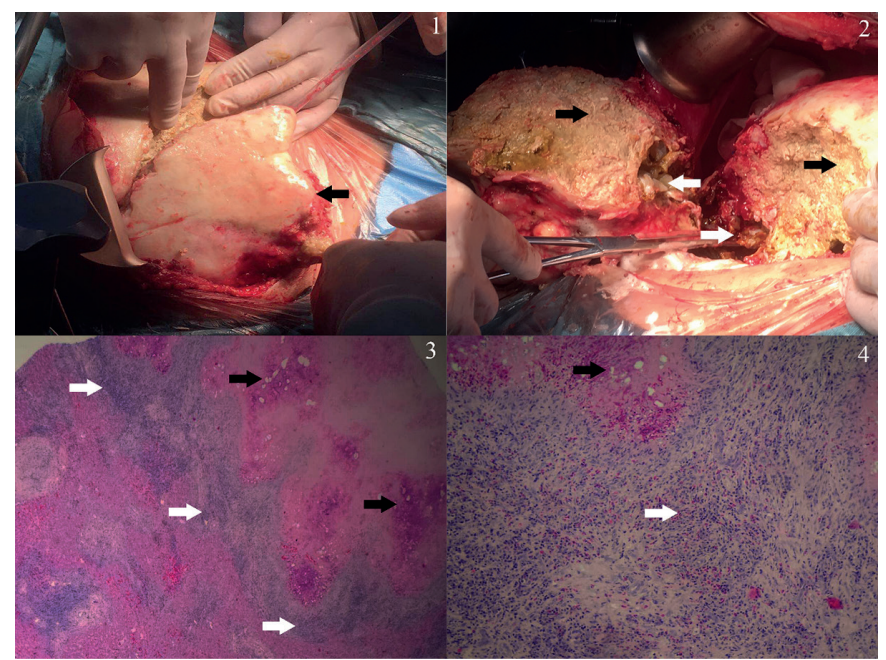

FIGURE B: Surgical photographs. (1): The black arrows indicate hepatic alveolar echinococcosis lesions. (2): The white arrows indicate hepatic cystic echinococcosis lesions. Hematoxylin and eosin staining. (3): The black and white arrows indicate the lesion and inflammatory response zones, respectively (original magnification $\times 10$ ). (4): The black and white arrows indicate the lesion and inflammatory response zones, respectively (original magnification $\times 100$ ). 
of the preoperative CT images verified that the lesions located in segment 3 and near the first hepatic portal area might have been caused by complete calcification of cystic echinococcosis $(\mathrm{CE} 4 \text { or CE5) })^{3}$. Although the probability of a mixed infection with hepatic cystic and alveolar echinococcosis is only $1 / 10^{5}$, the consequences of intraoperative fluid leakage cannot be ignored.

\section{Institutional review board statement}

The study was reviewed and approved by the local ethics committee (P-SL-2018005).

\section{Informed consent statement}

All study participants, or their legal guardian, provided informed written consent before study enrollment.

\section{Conflict of Interest}

The authors declare that there is no conflict of interest.

\section{Financial Support}

This study was supported by the Science and Technology Major Project of Qinghai Provincial Science and Technology Department (2016-SF-A5).

\section{REFERENCES}

1. Kern P, Wen H, Sato N, Vuitton DA, Gruener B, Shao Y, et al. WHO classification of alveolar echinococcosis: principles and application. Parasitol Int. 2006; 55(Suppl):S283-7.

2. Brunetti E, Kern P, Vuitton D A. Expert consensus for the diagnosis and treatment of cystic and alveolar echinococcosis in humans. Acta Trop. 2010; 114(1):1-6.

3. WHO Informal Working Group. International classification of ultrasound images in cystic echinococcosis for application in clinical and field epidemiological settings. Acta Trop. 2003;85(2),253-61. 